\title{
Structural Investigation of the (010) Surface of the $\mathrm{Al}_{13} \mathrm{Fe}_{4}$ Catalyst
}

\author{
J. Ledieu, ${ }^{1, *}$ É. Gaudry, ${ }^{1}$ L. N. Serkovic Loli, ${ }^{1}$ S. Alarcón Villaseca, ${ }^{1}$ M.-C. de Weerd, ${ }^{1}$ \\ M. Hahne, ${ }^{2}$ P. Gille, ${ }^{2}$ Y. Grin, ${ }^{3}$ J.-M. Dubois, ${ }^{1}$ and V. Fournée ${ }^{1}$ \\ ${ }^{1}$ Institut Jean Lamour (UMR7198 CNRS, Université de Lorraine), Parc de Saurupt, 54011 Nancy Cedex, France \\ ${ }^{2}$ Department of Earth and Environmental Sciences, Crystallography Section, Ludwig-Maximilians-Universität München, \\ Theresienstrasse 41, D-80333 München, Germany \\ ${ }^{3}$ Max-Planck-Institut für Chemische Physik fester Stoffe, Nöthnitzer Strasse 40, 01187 Dresden, Germany
}

(Received 9 November 2012; published 15 February 2013)

\begin{abstract}
We have investigated the structure of the $\mathrm{Al}_{13} \mathrm{Fe}_{4}(010)$ surface using both experimental and ab initio computational methods. The results indicate that the topmost surface layers correspond to incomplete puckered $(P)$ planes present in the bulk crystal structure. The main building block of the corrugated termination consists of two adjacent pentagons of $\mathrm{Al}$ atoms, each centered by a protruding $\mathrm{Fe}$ atom. These motifs are interconnected via additional $\mathrm{Al}$ atoms referred to as "glue" atoms which partially desorb above $873 \mathrm{~K}$. The surface structure of lower atomic density compared to the bulk $P$ plane is explained by a strong $\mathrm{Fe}-\mathrm{Al}-\mathrm{Fe}$ covalent polar interaction that preserves intact clusters at the surface. The proposed surface model with identified Fe-containing atomic ensembles could explain the $\mathrm{Al}_{13} \mathrm{Fe}_{4}$ catalytic properties recently reported in line with the site-isolation concept [M. Armbrüster et al., Nat. Mater. 11, 690 (2012)].
\end{abstract}

DOI: 10.1103/PhysRevLett.110.076102

Complex metallic alloys (CMAs) like quasicrystals and approximants are being considered as low-cost alternative materials for heterogeneous catalysis [1-6]. It relies on the so-called site-isolation concept in which the catalytic performance of a material is ascribed to small and wellseparated atomic ensembles containing an active transition metal (TM) element at the crystal surface [7]. Such atomic ensembles must be stable under reaction conditions, which in turn depend on the chemical bonding and the crystal structure of the intermetallic compound. $\mathrm{Al}_{13} \mathrm{Fe}_{4}$ has been identified recently as one such promising candidate for the heterogeneous hydrogenation catalysis. The $\mathrm{Al}_{13} \mathrm{Fe}_{4}(010)$ surface tested both under ultrahigh vacuum and under reaction conditions using $\mathrm{x}$-ray photoelectron spectroscopy measurements remains unaltered under acetylene hydrogenation conditions (no elemental iron formation). Although a partial oxidation is present, a sharp low energy electron diffraction pattern is initially observed on the (010) surface [1]. Quantum chemical calculations indicate strongly bounded $\mathrm{Fe}-\mathrm{Al}-\mathrm{Fe}$ groups encapsulated in cavities formed by the structurally complex Al network, which are believed to play an important role in the catalytic performance of this material. At this stage, it is not clear how such covalent interactions will influence the surface structure of the $\mathrm{Al}_{13} \mathrm{Fe}_{4}$ compounds. In this Letter, we investigate the structure of $\mathrm{Al}_{13} \mathrm{Fe}_{4}$ under ultrahigh vacuum and identify well-separated $\mathrm{Fe}$-containing atomic ensembles in line with the site-isolation concept. The resulting surface structure is a direct manifestation of the strong Fe-Al-Fe covalent interaction in the bulk structure, preserved up to the topmost surface layer and thermodynamically stable under the experimental conditions used in the present study.
PACS numbers: 68.35.bd, 68.37.Ef, 71.15.Mb, 71.20.Be

The monoclinic crystal structure of $\mathrm{Al}_{13} \mathrm{Fe}_{4}$ belongs to the space group $C 2 / m$ [8]. It is composed of 102 atoms (78 Al and $24 \mathrm{Fe}$ atoms) per unit cell with the following lattice parameters: $a=15.492 \AA, b=8.078 \AA$, and $c=$ $12.471 \AA$, with $\beta=107.69^{\circ}$ (Pearson symbol $m C 102$ ). As a four-layer approximant to the decagonal quasicrystal, its structure is commonly described as a stacking of flat $(F)$ and puckered $(P)$ layers with a pseudotenfold $(p 10 f)$ symmetry in a $F_{0.0} P_{0.25} F_{0.5} P_{0.75}$ sequence (the number stands for the $y$ coordinate) along the [010] direction. The $P_{0.25}$ and $P_{0.75}$ are mirrored against the $F_{0.5}$ layer. Connecting $\mathrm{Fe}$ atoms in the $F$ layers $(17 \mathrm{Al} / 8 \mathrm{Fe})$ leads to a tiling composed of rhombi and two types of pentagons, differentiated by their inner decoration with $\mathrm{Al}$ atoms. The $P$ layers $(22 \mathrm{Al} / 4 \mathrm{Fe})$ are described by elongated and distorted hexagons using the same procedure. The atomic structure of the $P$ layers can alternatively be interpreted as consisting of pentagons and additional $\mathrm{Al}$ atoms dubbed "glue" atoms [see Fig. 1(a)]. The individual Al pentagons are paired to form a bipentagonal motif. The Fe atoms are located slightly below (buried) or above (protruding) the mean plane of the pentagons. The individual Fe-centered Al pentagon corresponds to either the bottom or the top cap of the "pentagonal bipyramid" (PB) clusters suggested to describe geometrically the $\mathrm{Al}_{13} \mathrm{Fe}_{4}$ bulk structure [8-10]. Thus, the crystal structure has been recently interpreted as a cage one based on quantum chemical calculations $[11,12]$. As shown in Fig. 1(b), the chemical bonding analysis results in the interpretation of the $\mathrm{Al}_{13} \mathrm{Fe}_{4}$ as a three-dimensional $\mathrm{Al}$ framework where elongated cavities (host cage) encapsulate nearly linear $\mathrm{Fe}-\mathrm{Al}-\mathrm{Fe}$ groups (guest). While the bonding of the $\mathrm{Fe}-\mathrm{Al}-\mathrm{Fe}$ group is 


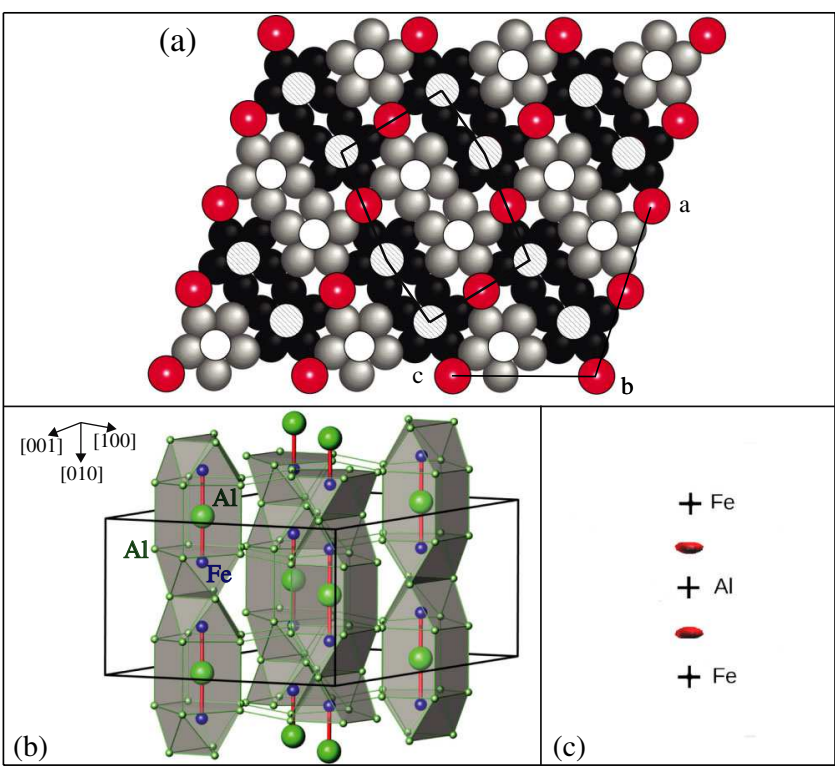

FIG. 1 (color online). (a) Representation of a $P$ layer present in the bulk crystal structure of $\mathrm{Al}_{13} \mathrm{Fe}_{4}$. The black and light gray spheres correspond to $\mathrm{Al}$ atoms forming bipentagonal motifs. The red (dark gray) spheres are associated with Al glue atoms. $\mathrm{Fe}$ atoms are located above (hatched circles) and below (empty circles) the mean $P$ plane position. An irregular elongated hexagon is drawn by connecting protruding Fe atoms. (b) 3D view of the crystal structure where both the nearly linear Fe-Al-Fe groups and the cages are displayed. (c) Isosurface of the deformation density $\left(\rho_{\text {def }}=0.078 e / \AA^{3}\right)$.

directional or covalent-this is highlighted by the enhanced deformation density along the Fe-Al-Fe bonds [Fig. 1(c)] - the ionic-type interaction between the guest and the host is nondirectional and probably weaker. Here, we investigate the influence of this specific bonding on the $p 10 f \mathrm{Al}_{13} \mathrm{Fe}_{4}(010)$ surface structure.

The single crystal of $\mathrm{Al}_{13} \mathrm{Fe}_{4}$ has been grown using the Czochralski method from an Al-rich solution [13], aligned by back reflection Laue $\mathrm{x}$-ray diffraction, cut perpendicular to its [010] direction, and polished down to $1 / 4 \mu \mathrm{m}$ diamond paste prior to insertion under ultrahigh vacuum conditions. Then, the preparation of the $\mathrm{Al}_{13} \mathrm{Fe}_{4}(010)$ surface consists of cycles of $\mathrm{Ar}^{+}$ion sputtering followed by annealing between 873 and $993 \mathrm{~K}$ for $90 \mathrm{~min}$. The temperature is measured using an infrared optical pyrometer (emissivity set to 0.35 ).

A $(1 \times 1)$ low energy electron diffraction pattern is observed after annealing the sample to $873 \mathrm{~K}$ [Fig. 2(a)]. The surface unit mesh is oblique with an angle of $107 \pm 1^{\circ}$ and with a lattice dimension ratio $\left(\frac{a}{c}=1.24\right)$ in accordance with those reported for the monoclinic $\mathrm{Al}_{13} \mathrm{Fe}_{4}$ phase [8]. In addition to the $(1 \times 1)$ pattern, there are fainter diffraction spots which are attributed to twinned domains present at the sample surface. Planar defects such as twins are intrinsic to the $\mathrm{Al}_{13} \mathrm{TM}_{4}$ compounds [14,15]. Angle-dependent $\mathrm{x}$-ray photoelectron spectroscopy measurements, performed from

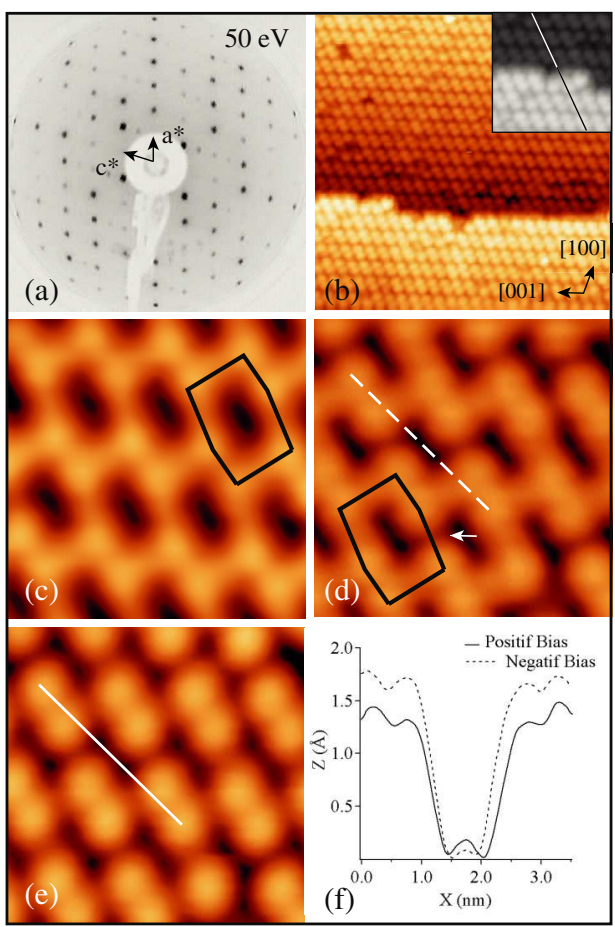

FIG. 2 (color online). (a) Low energy electron diffraction pattern of the $\mathrm{Al}_{13} \mathrm{Fe}_{4}(010)$ surface annealed to $873 \mathrm{~K}$. (b) STM image $\left(30 \times 30 \mathrm{~nm}^{2}\right)$ showing two terraces separated by a unique step height. Inset: $10 \times 10 \mathrm{~nm}^{2}$ region of Fig. 2(b). (c) $5 \times 5 \mathrm{~nm}^{2}$ STM image $\left(V_{b}=-1.05 \mathrm{~V}, I_{t}=0.5 \mathrm{nA}\right)$ obtained for a sample annealed to $873 \mathrm{~K}$. Connecting features of high contrast lead to the description of the structure by an irregular elongated hexagon as on Fig. 1(a). (d), (e) $5 \times 5 \mathrm{~nm}^{2}$ STM images acquired for the same region at (d) $V_{b}=-0.6 \mathrm{~V}$ and (e) $V_{b}=+1.0 \mathrm{~V}$ on the sample annealed to $993 \mathrm{~K}$. The white arrow outlines a missing glue atom, resulting in a lower contrast in between two vertices of the hexagon. The white lines on both images correspond to the 1D plot profiles presented in (f).

a $0^{\circ}$ to $60^{\circ}$ takeoff angle to increase the surface sensitivity, demonstrate that surface segregation can be disregarded for the $p 10 f$ surface. Following these results, the surface terminations should be related to $P$ and/or $F$ layers present in the bulk structure.

A scanning tunneling microscopy (STM) image [Fig. 2(b)] shows two consecutive terraces separated by a step height equal to half the lattice parameter $(b / 2)$ along the [010] direction. This is the unique step height measured at the $\mathrm{Al}_{13} \mathrm{Fe}_{4}(010)$ surface. These measurements imply that only one type of plane ( $P$ or $F$ ) should be present at the surface. This STM image exhibits motifs that are composed of two adjacent protrusions. The latter are reminiscent of what has been reported on the $\mathrm{Al}_{13} \mathrm{Co}_{4}(100)$ surface and then associated to bipentagonal motifs present on the $P$ layers [16-18]. For the monoclinic $\mathrm{Al}_{13} \mathrm{Fe}_{4}(010)$ system, similar bipentagonal motifs [gray or black in Fig. 1(a)] are aligned along the [101] direction. From $P_{0.25}$ to $P_{0.75}$, each bipentagonal motif is shifted by $7.78 \AA$ along an axis which is $5.21^{\circ}$ off the [100] direction. This is equivalent to a 
mutual exchange of bipentagonal motifs of one type by the other set, i.e., the gray (black) pentagons switch to black (gray) with protruding (buried) Fe atoms. This structural difference leads to an apparent shift of the lines propagating in the [101] direction between both the $P_{0.25}$ and the $P_{0.75}$ layers. This displacement is indeed observed experimentally in the inset of Fig. 2(b), where the line bisecting the protrusions on the upper terrace appears in between the motifs on the lower terrace. This effect by itself cannot be explained if the $F$ layers are the surface terminations because they are symmetrically equivalent.

High-resolution STM images show that the surface structure evolves with the annealing temperature [Figs. 2(c) and 2(d)]. At $873 \mathrm{~K}$, it can be described by a tiling based on irregular elongated hexagons, obtained by joining areas of bright contrast. Both the orientation and dimensions of this pattern are identical to the one drawn on Fig. 1(a). At 993 K, contrast discontinuities along the edges of the irregular hexagons [see the arrow in Fig. 2(d)] are commonly observed. Figures 2(d) and 2(e) exhibit STM images of opposite bias recorded exactly for the same region on the terrace. At positive bias, pairs of bright spots dominate the STM images, while, at negative bias, bright irregular elongated hexagons are visible. This bias dependency is expected from the asymmetric shape of the electronic density of states of $\mathrm{Al}_{13} \mathrm{Fe}_{4}$ [see Fig. 4(left)] [19]. Figure 2(f) shows two 1D plot profiles recorded along the lines drawn on Figs. 2(d) and 2(e). Depending on the STM tip polarity, a substantial height difference is measured over the same structural features. Nevertheless, both profiles exhibit a similar shape characterized by a deep trough. The peak-to-peak height difference is respectively equal to $1.47 \AA$ (solid line) and $1.77 \AA$ (dashed line). The position of this depression suggests that the gray bipentagonal motifs displayed in Fig. 1(a) have desorbed from the topmost layer (already gone at $873 \mathrm{~K}$ ). At this stage, it is important to recall that the $z$ position of the gray pentagons is higher than that of the black one by $0.48 \AA$ on $P$ bulk planes. A complete desorption of one set of pentagons along with their respective central $\mathrm{Fe}$ atoms should expose the $F$ plane that is underneath to the surface. From previous studies on quasicrystal and approximant surfaces, an inward relaxation between the two topmost surface layers is expected. This effect has been estimated here to $\sim 4 \%$ between the incomplete termination and the subsurface layer from the following calculations; i.e., it is consistent with the measured profiles displayed in Fig. 2(f) $[18,20,21]$. The intensity increase in the trough center on both profiles (even stronger at positive bias) could be attributed to the contribution of two Fe atoms which belong to the $F$ layer that is underneath.

Complementarily to this experimental approach, $a b$ initio calculations have been performed within the density functional theory framework using the projectoraugmented wave method [22,23] within the generalized gradient approximation of Perdew, Burke, and Ernzerhof
[24,25]. After relaxation, the resulting bulk lattice parameters are $a=15.43 \AA, b=8.03 \AA$, and $c=12.43 \AA$ $\left(\beta=104^{\circ}\right)$. Several surface models have been derived by considering the above observations. They are all built starting from bulk truncation at the $P$ plane. The eightlayer-thick asymmetric slabs are constructed with atoms of the four bottom layers being fixed to their bulk positions while the remaining atoms are allowed to relax.

The first model preserves a complete $P$ layer at the surface. Additional models are built from an incomplete $P$ layer where one set of bipentagonal motifs has been removed. Hence, only intact PB clusters are preserved at the surface. Further differentiations among these models rely on the occupancy of the Al glue atoms connecting the bipentagonal motifs. Contrary to the complete $P$ layer, a qualitative good agreement is obtained between the simulated STM images of the incomplete $P$ terminations and the experimental ones [Figs. 3(b)-3(f)]. The pairs of adjacent bright spots on the experimental image [Fig. 2(e)] can then be attributed to bipentagonal Fe-protruded $\mathrm{Al}$ motifs. Similarly, the structural evolution with the annealing temperature highlighted in Figs. 2(c) and 2(d) is now understood by the consecutive desorption of the surface $\mathrm{Al}$ glue atoms. The total energy calculations demonstrate that the stability of the incomplete $P$ layers is reduced incrementally by $\simeq 0.6 \mathrm{eV}$ (calculated with $\mu_{\mathrm{Al}}=\mu_{\mathrm{Al}}^{\text {bulk }}$ ) every time an $\mathrm{Al}$ glue atom is removed. In addition, the simulated STM images [Figs. 3(c) and 3(d)] reproduce the bias

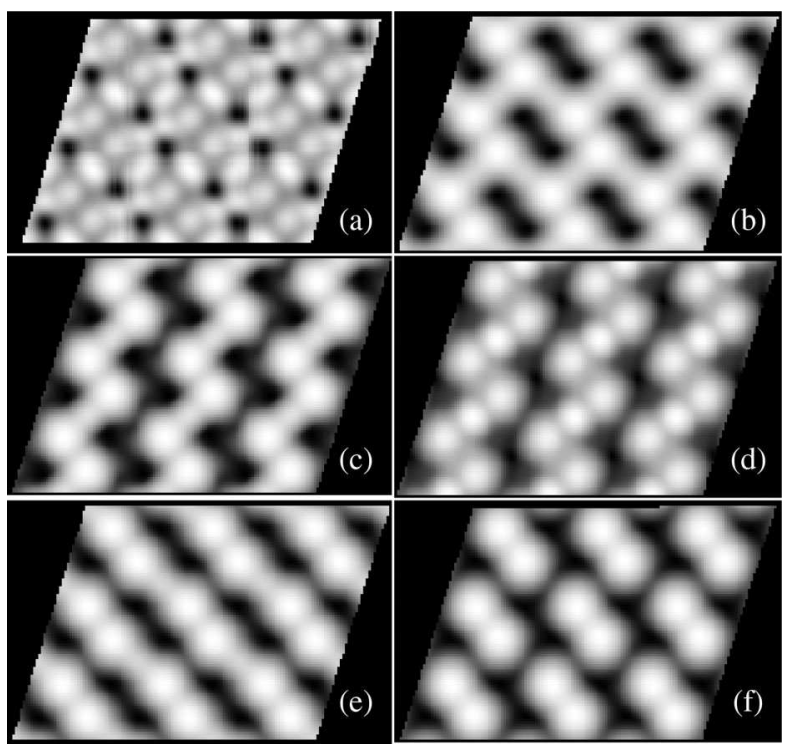

FIG. 3. Simulated constant current STM images of $\mathrm{Al}_{13} \mathrm{Fe}_{4}$ $(3 a \times 3 c)$ for (a) a complete $P$ layer, (b) an incomplete $P$ layer that preserves the PB clusters at the surface $\left(V_{\text {bias }}=-1 \mathrm{~V}\right)$. (c), (d) The same model as (b) with only one surface Al glue atom [(c) $V_{\text {bias }}=-1 \mathrm{~V}$; (d) $V_{\text {bias }}=+1 \mathrm{~V}$ ]. (e) The same model as (b) with the other surface $\mathrm{Al}$ glue atoms $\left(V_{\text {bias }}=-1 \mathrm{~V}\right)$. (f) The same model as (b) with no Al glue atoms $\left(V_{\text {bias }}=-1 \mathrm{~V}\right)$. 


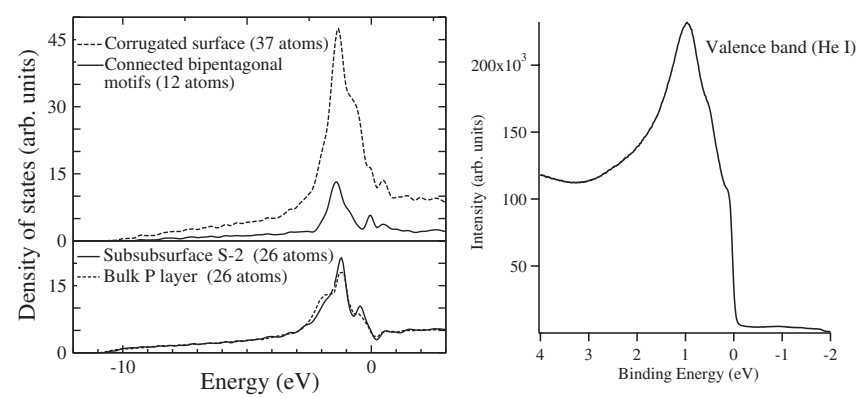

FIG. 4. Left: Electronic DOS of the surface and subsurface layers of $\mathrm{Al}_{13} \mathrm{Fe}_{4}$. Right: Ultraviolet photoelectron spectroscopy valence-band spectrum of the clean surface.

dependency observed experimentally along with adjacent bright spots at positive bias.

The layer-resolved densities of states (DOS) for the model presented in Fig. 3(f) are shown in Fig. 4. The DOS are all dominated by the Fe atoms' $d$ states which extend up to $E_{F}$. For the incomplete surface layer $(S)$, two major contributions can be located, respectively, at $-1.4 \mathrm{eV}$ and at $E_{F}$. It is reasonable to expect that the close vicinity of these states to $E_{F}$ should play a major role for the surface chemical reactivity. Additional contributions close to $E_{F}$ are present when combining both $S$ and $S-1$ layers (referred to as a corrugated surface), i.e., the DOS of the two planes leading to the corrugated surface. The main features emerging from these calculations replicate the ultraviolet photoelectron spectroscopy spectrum recorded at normal emission.

Although both intermetallic compounds share similar basic building blocks, the structural model obtained for the $\mathrm{Al}_{13} \mathrm{Fe}_{4}(010)$ surface differs from the experimentally determined $\mathrm{Al}_{13} \mathrm{Co}_{4}(100)$ surface in the sense that $\mathrm{Co}$ atoms protruding at the center of $\mathrm{Al}$ bipentagonal motifs are missing [18]. However, numerical cleavage carried out on the $\mathrm{Al}_{13} \mathrm{Co}_{4}(100)$ surface using ab initio calculations leads to a similar corrugated surface with Co-protruded elements [17]. An explanation for this experimental discrepancy may be found when analyzing the TM-Al-TM bonding strength between the two systems. To this end, the formation energy of (i) a single $\mathrm{Al}$ vacancy $\left(E_{f}^{\mathrm{vac}-\mathrm{Al}}\right)$, (ii) a single TM vacancy $\left(E_{f}^{\mathrm{vac}-\mathrm{TM}}\right)$, and (iii) a double Al-TM vacancy $\left(E_{f}^{\mathrm{vac}-\mathrm{AlTM}}\right)$ have been calculated, all part of the TM-Al-TM group. The strength of the binding is then evaluated as follows:

$$
E_{\text {strength }}^{\mathrm{Al}-\mathrm{TM}}=E_{f}^{\mathrm{vac}-\mathrm{AlTM}}-\left(E_{f}^{\mathrm{vac}-\mathrm{Al}}+E_{f}^{\mathrm{vac}-\mathrm{TM}}\right) .
$$

The calculation gives $E_{\text {strength }}^{\mathrm{Al}-\mathrm{Co}}=-0.31 \mathrm{eV}$ and $E_{\text {strength }}^{\mathrm{Al}-\mathrm{Fe}}=$ $-0.66 \mathrm{eV}$. The negative value of $E_{\text {strength }}$ reflects the covalent character of the Al-TM bonding. The stronger Al-Fe bonding could explain the structural differences observed between these two compounds, with intact clusters being preserved at the $\mathrm{Al}_{13} \mathrm{Fe}_{4}(010)$ surface.
As in previous studies on CMAs, there is neither surface reconstruction (no modification of the reciprocal lattice) nor chemical segregation on the surface. Out of the two possible terminating planes, the puckered layers represent the densest and the most Al-rich configuration. Hence, the selection of $P$ layers as surface termination is not unexpected and follows the trend established on other CMA phases. However, the $\mathrm{Al}_{13} \mathrm{Fe}_{4}(010)$ surface is not obtained from a simple bulk truncation. The topmost surface layer consists of an incomplete $P$ layer where only one set of bipentagonal motifs centered by protruding $\mathrm{Fe}$ atoms is preserved along with the glue $\mathrm{Al}$ atoms. The latter start to partially desorb upon annealing the sample to temperatures higher than $873 \mathrm{~K}$. This mechanism results in a partial exposition of the underneath $F$ plane. To understand the desorption and a fortiori stability of certain entities within the layer, the chemical bonding has to be taken into account. Only intact bipyramid clusters (preserving the $\mathrm{Fe}-\mathrm{Al}-\mathrm{Fe}$ group [11]) remain stable at the $\mathrm{Al}_{13} \mathrm{Fe}_{4}(010)$ surface. Dissected bipyramidal clusters [gray bipentagonal motifs in Fig. 1(a)] distributed across the $P$ layer are unstable entities. This desorption phenomenon is not observed on the fivefold icosahedral and tenfold decagonal quasicrystalline surfaces, although highly symmetric clusters are dissected at different heights to generate atomic flat surfaces [26]. The catalytic activity of $\mathrm{Al}_{13} \mathrm{Fe}_{4}$ relies on the site-isolated concept where stable isolated $\mathrm{Fe}$ atoms are contained in ensembles. Following previous theoretical work on the $\mathrm{Al}_{13} \mathrm{Co}_{4}(100)$ surface [5,6], we suggest that the active sites are the isolated Fe atoms protruding above pentagonal motifs. Consequently, the catalytically active ensembles may be identified here as the stable $\mathrm{Al}_{5} \mathrm{Fe}$ complex decorating the $\mathrm{Al}_{13} \mathrm{Fe}_{4}(010)$ surface.

The Agence Nationale de la Recherche (ANR-08-Blan0041-01) and the Région Lorraine are thanked for their financial support. R. D. Diehl and K. Pussi are acknowledged for fruitful discussions. This work was supported by the HPC resources of the IDRIS under Allocation No. 99642 made by GENCI. This work is a result of cooperation within the European C-MAC.

*Corresponding author. julian.ledieu@ijl.nancy-universite.fr

[1] M. Armbrüster, K. Kovnir, M. Friedrich, D. Teschner, G. Wowsnick, M. Hahne, P. Gille, L. Szentmiklósi, M. Feuerbacher, M. Heggen, F. Girgsdies, D. Rosenthal, R. Schlögl, and Yu. Grin, Nat. Mater. 11, 690 (2012).

[2] T. Tanabe, S. Kameoka, and A. P. Tsai, Catal. Today 111, 153 (2006).

[3] M. Armbrüster, K. Kovnir, Yu. Grin, R. Schlögl, P. Gille, M. Heggen, and M. Feuerbacher, European Patent No. EP09157875.7 2009.

[4] T. Tanabe, S. Kameoka, and A. P. Tsai, Appl. Catal., A 384, 241 (2010). 
[5] M. Armbrüster, K. Kovnir, Yu. Grin, and R. Schlögl, in Complex Metallic Alloys: Fundamentals and Applications, edited by J.-M. Dubois and E. Belin-Ferré (Wiley-VCH, Weinheim, 2011), p. 385.

[6] M. Krajčí and J. Hafner, J. Catal. 278, 200 (2011).

[7] K. Kovnir, M. Armbrüster, D. Teschner, T. V. Venkov, F. C. Jentoft, A. Knop-Gericke, Yu. Grin, and R. Schlögl, Sci. Tech. Adv. Mater. 8, 420 (2007).

[8] J. Grin, U. Burkhardt, M. Ellner, and K. Peters, Z. Kristallogr. 209, 479 (1994).

[9] P. J. Black, Acta Crystallogr. 8, 43 (1955).

[10] C. L. Henley, J. Non-Cryst. Solids 153-154, 172 (1993).

[11] Yu. Grin, B. Bauer, U. Burkhardt, R. Cardoso-Gil, J. Dolinšek, M. Feuerbacher, P. Gille, F. Haarmann, M. Heggen, P. Jeglič, M. Müller, S. Paschen, W. Schnelle, and S. Vrtnik, EUROMAT 2007: Proceedings of the European Congress on Advanced Materials and Processes, Nürnberg, Germany, 2007 (Deutsche Gesellschaft für Materialkunde, Frankfurt, 2007).

[12] P. Jeglič, S. Vrtnik, M. Bobnar, M. Klanjsek, B. Bauer, P. Gille, Yu. Grin, F. Haarmann, and J. Dolinsek, Phys. Rev. B 82, 104201 (2010).

[13] P. Gille and B. Bauer, Cryst. Res. Technol. 43, 1161 (2008).

[14] U. Burkhardt, Ph.D. thesis, University of Stuttgart, 1993.

[15] K. Saito, K. Sugiyama, and K. Hiraga, Mater. Sci. Eng. 294-296, 279 (2000).
[16] R. Addou, E. Gaudry, Th. Deniozou, M. Heggen, M. Feuerbacher, P. Gille, Yu. Grin, R. Widmer, O. Gröning, V. Fournée, J.-M. Dubois, and J. Ledieu, Phys. Rev. B 80, 014203 (2009).

[17] M. Krajčí and J. Hafner, Phys. Rev. B 84, 115410 (2011).

[18] H. Shin, K. Pussi, É. Gaudry, J. Ledieu, V. Fournée, S. A. Villaseca, J.-M. Dubois, Yu. Grin, P. Gille, W. Moritz, and R. D. Diehl, Phys. Rev. B 84, 085411 (2011).

[19] M. Mihalkovič and M. Widom, Phys. Rev. B 75, 014207 (2007).

[20] M. Gierer, M. A. Van Hove, A. I. Goldman, Z. Shen, S.-L. Chang, C. J. Jenks, C.-M. Zhang, and P. A. Thiel, Phys. Rev. Lett. 78, 467 (1997).

[21] N. Ferralis, K. Pussi, E. J. Cox, M. Gierer, J. Ledieu, I. R. Fisher, C. J. Jenks, M. Lindroos, R. McGrath, and R. D. Diehl, Phys. Rev. B 69, 153404 (2004).

[22] P. E. Blöchl, Phys. Rev. B 50, 17953 (1994).

[23] G. Kresse and D. Joubert, Phys. Rev. B 59, 1758 (1999).

[24] J. P. Perdew, K. Burke, and M. Ernzerhof, Phys. Rev. Lett. 77, 3865 (1996).

[25] J. P. Perdew, K. Burke, and M. Ernzerhof, Phys. Rev. Lett. 78, 1396 (1997).

[26] R. McGrath, J. A. Smerdon, H. R. Sharma, W. Theis, and J. Ledieu, J. Phys. Condens. Matter 22, 084022 (2010). 